\title{
Chromosome Arm
}

National Cancer Institute

\section{Source}

National Cancer Institute. Chromosome Arm. NCI Thesaurus. Code C13355.

Under the microscope chromosomes appear as thin, thread-like structures. They all have a short arm and long arm separated by a primary constriction called the centromere. The short arm is designated as $p$ and the long arm as $\mathrm{q}$. 